\title{
An Interferometric Technique for Synthetic Aperture Ground-Penetrating Radar
}

\author{
Carl Leuschen, Nathan Goodman, Chris Allen, Richard Plumb \\ The University of Kansas, Remote Sensing Laboratory \\ 2291 Irving Hill Road, Lawrence, KS 66044 \\ TEL: 913/864-3017 , FAX: 913/864-7789 , E-mail: callen@eecs.ukans.edu
}

\begin{abstract}
Many synthetic aperture radar (SAR) algorithms for ground-penetrating radar involve making measurements along a single path and using the collected data to form a twodimensional image of the scattering object. In the calculation of these images, certain assumptions are made about the scattering objects; mainly, that the reflections are occurring directly below the antenna location, which is not always the case. By applying interferometric techniques to SAR-based images, it is possible to construct an image of the scattering object that contains all three dimensions rather than only two, eliminating the need for these assumptions. This paper proposes a method of combining radar interferometry with SAR-based ground-penetrating radar. The methodology used is to expand several two-dimensional images, each calculated from measurements collected along single paths, into a threedimensional image of the scattering object through the use of radar interferometry. This process involves superimposing two complex two-dimensional images and using the information found in the phase differences of corresponding locations from each image to calculate the location of the object in the dimension not contained within the previous two-dimensional images. This method is tested using both computer-simulated data and also data collected from the measurements of objects buried in sand.
\end{abstract}

\section{INTRODUCTION}

Currently, most interferometric applications consist of topographic mapping from data collected either from aircraft or satellite. This paper uses the techniques developed for these applications and applies them to the case of subsurface imaging in which the reflections occur much closer to the radar antennas. The primary objective in the application of interferometry to ground-penetrating radar is to expand the two-dimensional images collected using SAR processing into three dimensions. Since two-dimensional images contain ambiguities concerning the exact scatterer locations in real space, the need for an image of subsurface reflections containing all of the dimensions is essential in obtaining an accurate idea of what lies beneath the surface.

Although this technique illustrates a method to image a subsurface area, a prior knowledge of the velocity profile of the ground is required to obtain the correct distances from the time responses. Also, as with many imaging techniques, the reflections are assumed to originate from point scatterers. Due to these two assumptions, the main scope of this technique is for homogeneous ground with localized scatterers.

\section{DEVELOPMENT}

Through the application of interferometry to twodimensional synthetic aperture radar (SAR) images, the reflections of the scattering targets can be expressed with locations containing all three dimensions, eliminating any ambiguity found in the previous images. The process of interferometry involves examining the phase difference at corresponding locations of two two-dimensional SAR images in order to obtain information of the reflection's location in terms of the dimension not contained within the SAR images. The basic geometry for an interferometric application, as shown in Fig. 1, consists of two SAR images collected along a parallel path separated by a baseline, $B$. The goal is to generate an image in a vertical plane parallel to the SAR paths at a distance, $d$, from path 1 .

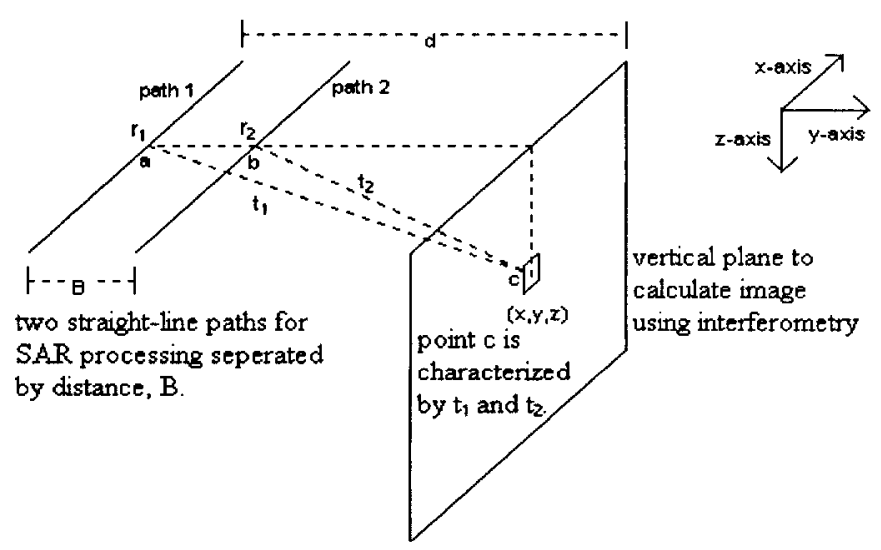

Fig. 1. The basic geometry for an interferometric application 
To demonstrate how interferometry is used to calculate images in terms of all three dimensions, the response due to a single point scatterer will be considered first, and then the existence of multiple scatterers is assumed to produce similar results due to superposition. However, by applying superposition, all the coupling effects between scatterers is ignored. Before applying interferometry, the images are expected to contain resolution in range and also in the same dimension of the two paths as a result of SAR processing. The images will be calculated in three dimensions by using the information found in the phase differences at corresponding locations along the two SAR paths to expand the range dimension into a depth, $z$, at a specific distance, $d$, resulting in a two-dimensional image at a constant distance from path 1 .

By considering two SAR images calculated from measurements collected along two parallel paths in the presence of a single scatterer, the time responses, $r_{1}$ and $r_{2}$, at corresponding locations along the path can be related by a time shift, $t_{s}$, due to the separation of the baseline, $B$. This time shift is represented in the frequency domain as a phase shift as shown by

$$
\begin{aligned}
& r_{1}(t)=r_{2}\left(t-t_{s}\right) \\
& R_{1}(f)=R_{2}(f) \exp \left(-j 2 \pi f t_{s}\right) .
\end{aligned}
$$

The relationship of the responses in the frequency domain provides the phase information, seen on the right-hand side of (2), as a direct result from the time shift in (1). Since this time shift is uniquely determined by the scatterer's location and also by the baseline, $B$, the phase information is used to determine the scatterer's depth for a given distance, $d$, from path 1.

By noticing that the scatterer's location is related to the time shift in (1), a response describing the relationship between $r_{1}$ and $r_{2}$ in terms of any time shift, $\tau$, is expressed by the correlation of $r_{1}$ and $r_{2}$, which is shown in both the time domain (3) and the frequency domain (4).

$$
\begin{aligned}
& r_{12}(\tau)=\sum_{k} r_{1}\left(t_{k}\right) r_{2}\left(t_{k}-\tau\right) \\
& r_{12}(\tau)=\frac{1}{N} \sum_{i=1}^{N} R_{1}\left(f_{i}\right) R_{2}^{*}\left(f_{i}\right) \exp \left(j 2 \pi f_{i} \tau\right) .
\end{aligned}
$$

In (4), $R^{*}$ represents the complex conjugate. Substituting the relationship of $R_{1}$ and $R_{2}$ for a single scatterer (2) into (4), $r_{12}$ can be shown to have a peak value occurring at a time shift, $t$, which corresponds to the scatterer's location.

$$
\begin{aligned}
r_{12}(\tau) & =\sum_{i=1}^{N} R_{2}\left(f_{i}\right) \exp \left(-j 2 \pi f_{i} t_{s}\right) R_{2}^{*}\left(f_{i}\right) \exp \left(j 2 \pi f_{i} \tau\right) \\
& =\sum_{i=1}^{N}\left|R_{2}\left(f_{i}\right)\right|^{2} \exp \left(j 2 \pi f_{i}\left(\tau-t_{s}\right)\right)
\end{aligned}
$$

Since the correlation response of $r_{1}$ and $r_{2}$, (3) and (4), contains peak values at time shifts corresponding to the locations of scatterers, by normalizing this response between zero and one, the correlation can be used to show the tendency for a scatterer to exist at any location that is characterized by a specific time shift, $\tau$.

$c_{12}(\tau)=\frac{r_{12}(\tau)}{\sqrt{r_{11}(0) r_{22}(0)}}$

An image of a vertical plane occurring at a distance, $d$, is now formed by combining the contributions of the two individual responses, $r_{1}$ and $r_{2}$, along with the response of their normalized correlation, $r_{12}$. As shown in the Fig. 1, every point in the imaging area can be characterized by two times, $\left(t_{1}, t_{2}\right)$, corresponding to the point's location relative to path 1 and path 2 .

$(x, y, z) \Rightarrow\left(t_{1}, t_{2}\right)$

Finally, an approximation to a specific location is calculated by scaling the average of the two responses at their corresponding times, $\left(t_{1}, t_{2}\right)$, by the value of the normalized correlation at the time shift, $\left(t_{1}-t_{2}\right)$.

$p(x, y, z)=c_{12}\left(t_{1}-t_{2}\right) \frac{1}{2}\left\{r_{1}\left(t_{1}\right)+r_{2}\left(t_{2}\right)\right\}$

By using this technique, an area spanned by two parallel SAR images can be described in all three dimensions.

\section{SIMULATION}

To demonstrate how interferometry can be used to expand two two-dimensional SAR images into three dimensions, a simple test configuration was simulated on a computer. With respect to Fig. 1, the configuration consists of two SAR paths separated by a baseline of one meter with 16 measurements collected along each path. The configuration also consists of $\backslash$ 


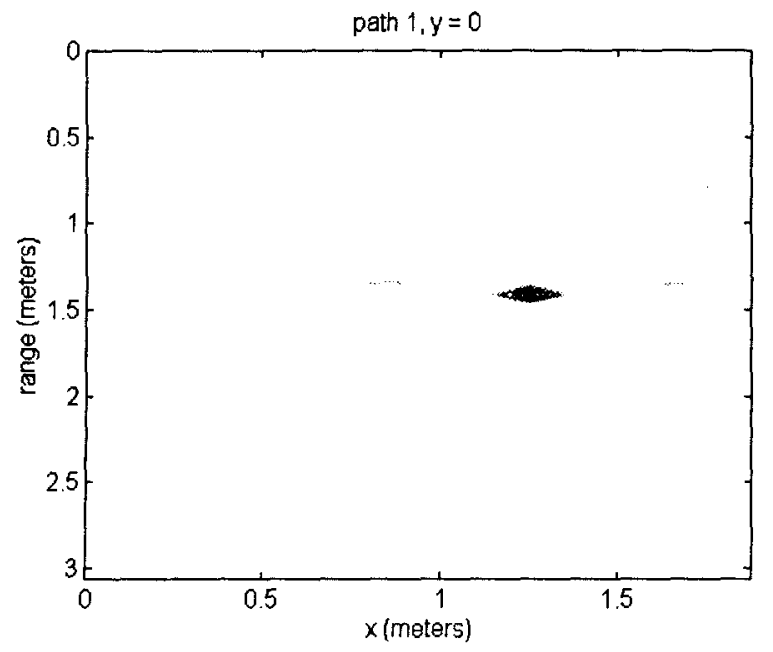

Fig. 2. SAR image along path 2

two scatterers located at coordinates $(1.25,1,1)$ and $(1.25,0,1.4142)$ such that the reflections appear at equal times from path 1. By using the difference between the two images calculated from measurements collected along the two paths, the single reflection occurring in image 1 is expanded into locations in three dimensions to describe scatterers located at two coordinates.

Fig. 2 and Fig. 3 are the SAR images calculated from the data simulated along two paths seperated by a distance of one meter. As expected there exists only one reflection in Fig. 2 since the scatterers were located equal distances from path 1 . By applying interferometry to the two images, the locations of the two scatterers were separated into their correct positions as shown in Fig. 4.

\section{DISCUSSION}

In this paper, a technique has been presented in which two two-dimensional SAR-based images are used in order to obtain an image containing all three dimensions, eliminating any assumptions or requirements concerning the imaging area. This technique has been tested from data simulated on a computer using phase propagation. For further verification, this technique will be tested using real data collected from measurements of objects buried in sand, which also meets the assumption of homogeneous ground with localized scatters.

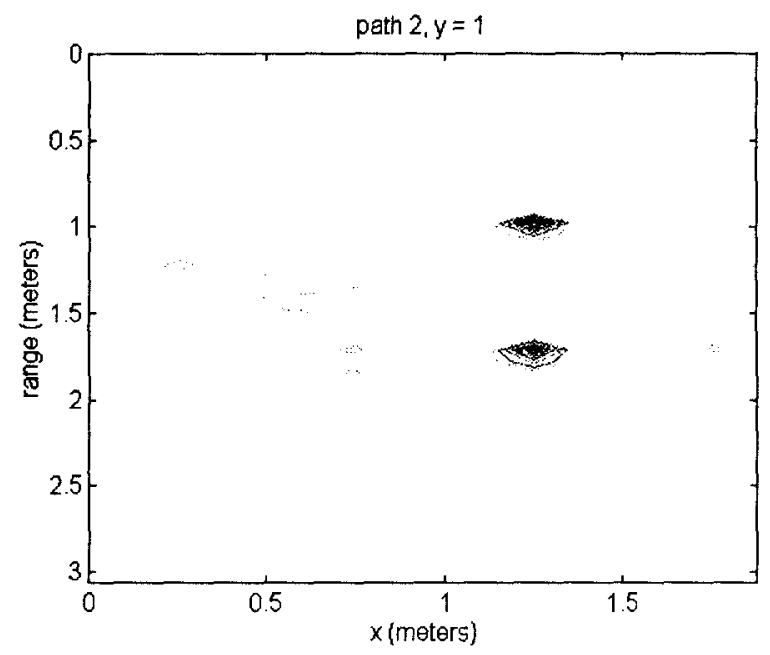

Fig. 3. SAR image along path 1

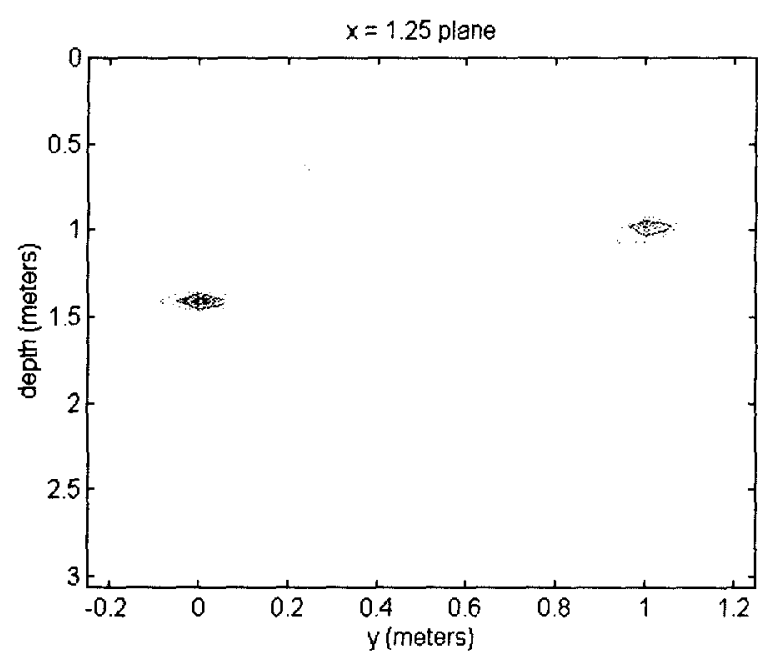

Fig. 4. Image calculated using interferometry

\section{REFERENCES}

[1] C. T. Allen, "Interferometric Synthetic Aperture Radar," IEEE Geoscience and Remote Sensing Newsletter, issue 96, pp. 6-13, September 1995.

[2] D. Massonnet and T. Rabaute, "Radar Interferometry: Limits and Potential," IEEE Trans. on Geoscience and Remote Sensing, vol. 31, no. 2, pp. 455-464, March 1993.

[3] A. Gunawardena and D. Longstaff, "A Matched Filter Based Synthetic Aperture Radar(SAR) Algorithm For Stepped Frequency Ground Penetrating Radar," IEEE International Radar Conference, pp. 239-243, 1995. 